\title{
Estudio descriptivo comparativo de competencia digital en docentes de tres instituciones educativas. Apurímac, 2021
}

Cindy Vílchez Ramírez

vilchez.cindy@gmail.com

https://orcid.org/0000-0001-7988-8580

Nicanor Piter Saavedra Carrión

nicanor.saavedra@ayacucho.edu.pe https://orcid.org/0000-0001-5827-9524

Gabriela Soledad Vásquez Urbina gvasquezur@ucvvirtual.edu.pe https://orcid.org/0000-0002-1286-344X

Mónica Patricia Vásquez Urbina mpvasquez20@ hotmail.com https://orcid.org/0000-0001-8258-2371

Evelyn Ucharima Huarcaya evelyn0001uh@gmail.com https://orcid.org/0000-0003-0150-0616

Miryam Gladys, Pineda Morán miryamgladys4164@gmail.com https://orcid.org/0000-0002-2307-9191

Dante Vidal, Coaguila Mayanaza davicom007@gmail.com https://orcid.org/0000-0002-4866-5277

\section{RESUMEN}

El presente trabajo tuvo como objetivo determinar y comparar el nivel de competencia digital en docentes de tres instituciones educativas de nivel secundario, de tipo aplicado, de enfoque cuantitativo, de método hipotético deductivo, de nivel descriptivo comparativo y de diseño no experimental, con corte transeccional, donde se consideró como población a 108 docentes de las tres instituciones educativas y mediante muestreo probabilístico estratificado se determinó la muestra conformada por 85 docentes, a quienes para recabar datos se les aplicó como instrumento un cuestionario, de 30 ítems, validado por opinión de experto y de 0,870 de índice de confiabilidad. Referente a los resultados, se evidencio que la significancia de la prueba de Kruskal-Wallis fue de 
0,072>0,05; además, se observó que la diferencia de rangos de la competencia digital de los docentes, no difiere en gran cantidad, por tanto, no se rechazó la hipótesis nula, concluyendo que no existen diferencias significativas en el nivel de competencia digital en docentes de tres instituciones educativas de nivel secundario.

Palabras clave: competencia digital; herramientas digitales; desempeño docente. 


\title{
Comparative descriptive study of digital competence in teachers of three educational institutions. Apurimac, 2021
}

\begin{abstract}
The objective of this study was to determine and compare the level of digital competence in teachers of three secondary level educational institutions, applied, quantitative approach, hypothetical deductive method, descriptive comparative level and nonexperimental design, with transectional cut, where 108 teachers from the three educational institutions were considered as the population, and by means of stratified probabilistic sampling, the sample was determined to be made up of 85 teachers, to whom a 30-item questionnaire was applied as an instrument to collect data, validated by expert opinion and with a reliability index of 0.870 . Regarding the results, it was found that the significance of the Kruskal-Wallis test was $0.072>0.05$; in addition, it was observed that the difference of ranks of the digital competence of the teachers did not differ greatly, therefore, the null hypothesis was not rejected, concluding that there are no significant differences in the level of digital competence in teachers of three educational institutions of secondary level.
\end{abstract}

Keywords: digital competence; digital tools; teaching performance

Artículo recibido: 15 noviembre. 2021 Aceptado para publicación: 10 diciembre 2021

Correspondencia: vilchez.cindy@gmail.com Conflictos de Interés: Ninguna que declarar 


\section{INTRODUCCIÓN}

En la actualidad, se ha incrementado la necesidad de desarrollar la competencia digital, por la masiva incorporación de herramientas digitales desde la instauración de la pandemia por el Covid-19, en Perú, arraigando variados factores de riesgo propios de las instituciones educativas que hacen complicada y difícil la ejecución de la práctica pedagógica docente al brindar el servicio educativo remoto a causa del aislamiento social dispuesto por el gobierno nacional (PCM, 2020); en tal sentido, Cruz y Carcausto-Calla (2020), dan a entender que esos factores son evidenciados al ejercer su trabajo docente, al no poseer algún conocimiento digital, al mostrar una actitud resilente o por la falta de conectividad, y en gran mayoría por economía escasa de los padres de familia.

Asimismo, la UNESCO (2021), ha monitoreado la influencia del Covid-19, en el sector educación de todo el mundo, estimando que un año luego de darse la alerta de su llegada, cerca de 800 millones de escolares, siguen afrontando interrupciones en su educación, al no poder asistir a la escuela o que las clases se den virtualmente recortando horas efectivas, instaurando en muchos países la educación virtual o también conocida como educación a distancia, en dos modalidades: sincrónica y asincrónica (Cantián, 2020), lo cual exige a los docentes desarrollar su competencia digital, ya que es tomada según Marza y Cruz (2018), como herramienta indispensable, cuyo propósito posibilita la adquisición de conocimiento, el cual ha cambiado el paradigma tradicional innovando el proceso de aprendizaje.

En América Latina, de la misma manera se ha visto afectado el sector educación, por optar también por el cierre de sus escuelas durante el mes de marzo del año 2020, y un año después sigue aún cerradas ya que el Covid-19, está manifestándose más agresivamente, en tal sentido, la UNESCO (2020), manifestó que el docente es considerado como mediador del saber, son los que tienen una relación formidable con los estudiantes, responsable de la educación de la futura sociedad y que en ellos recae dar continuidad a la educación en estos momentos difíciles, por tales motivos, países como Uruguay ya ha venido facilitando la implementación de herramientas digitales, en beneficio de todos los escolares, pioneros en la entrega de laptops a sus estudiantes, México, ha puesto sus esfuerzos en la telesecundaria, usando tecnología tradicional con alto impacto dirigido al sector poblacional en vulnerabilidad garantizando la equidad de acceso a la educación, Colombia, invierte en entornos virtuales, con miras al 
fortalecimiento de la competencia digital, en donde se facilita recursos ya digitalizados por grados de estudio, los cuales contienen diversas aplicaciones. Cabe señalar, que los países ya mencionados han afrontada este complicado momento adecuadamente, al brindar una educación no presencial adecuada, pero todo ello no daría resultados si el docente no se encuentra capacitado para el manejo de las herramientas tecnológicas.

En Perú, es preciso indicar que algo más del $70 \%$ de personas, están frente a la computadora muchas horas al día, pero se sabe que más del $20 \%$ no lo utilizaba para estudiar, sino para actividades asociadas al ocio. (INEI, 2019), de esa manera no se han logrado desarrollar la competencia digital, ni se le ha dado un fin educativo. Asimismo, para afrontar el cierre de las instituciones educativas, el Minedu ha lanzado la estrategia educativa Aprendo en Casa (MINEDU, 2020), con el objetivo de dar continuidad a la educación en el territorio nacional, pero dicha implementación ha desnudado deficiencias en el manejo de herramientas tecnológicas y en consecuencia se ha obtenido un desarrollo nulo de la competencia digital por parte de la comunidad educativa, resaltar que una quinta parte de docentes, no la ha adquirido sea por no tomarlas en cuenta en su práctica pedagógica o por tener un pensamiento tradicional.

En el departamento de Apurímac, la mayor cantidad de instituciones educativas están ubicadas en zonas cuyo grado de ruralidad se encuentra en uno, dos y tres, lo cual significa que se caracterizan por un escaso uso de recursos tecnológicos, existiendo una gran brecha digital que hasta el momento no se ha cerrado en su totalidad, además, dichas zonas no tienen conectividad fluida de TV, radio e internet, ya que al llover o nublarse ocasiona que la escasa señal se interrumpa, asimismo en la UGEL Chincheros, en el nivel secundario se cuenta con docentes de diferentes especialidades que en gran parte no se han familiarizado con el uso de recursos tecnológicos para mediar o retroalimentar la enseñanza remota, por tal motivo, surge la necesidad que los docentes desarrollen la competencia digital, ya que ello sería de gran ayuda porque facilitaría la comunicación con los estudiantes para brindarles una educación remota que favorezca el logro de competencias y que asegure la continuidad de la educación.

Por las razones anteriores se planteó el siguiente problema de investigación: ¿Cuál es el nivel de competencia digital en docentes de tres instituciones educativas de nivel secundario. Apurímac 2021?, a continuación, se planteó la hipótesis general: Existen diferencias significativas en el nivel de competencia digital en docente de tres 
instituciones educativas de nivel secundario. Apurímac 2021, finalmente, como objetivo general: Determinar y comparar el nivel de competencia digital en docentes de tres instituciones educativas de nivel secundario. Apurímac 2021.

Por otro lado, la teoría del presente estudio, consideró al paradigma como un modelo que posibilita la elaboración de trabajos de investigación (Pérez, 2015), compuesto por un sistema de postulados, principios, creencias e ideologías que precisan un camino organizado sobre el campo de la investigación de una comunidad científica, asimismo, en el campo educativo existen tres tipos de paradigma el "positivista, interpretativo y sociocrítico" (Gil et àl., 2017, p. 73), en tal sentido, el paradigma positivista o cuantitativo, fue seleccionado para el presente estudio, al facilitar el planteamiento de problemas de investigación y al constatar las hipótesis que fueron planteadas por medio del uso de instrumentos que fueron validados y evidenciado su confiabilidad, para que posteriormente los resultados obtenidos puedan generalizarse a toda la población.

Asimismo, la teoría del Conectivismo, propuesto por Siemens (2006), sustenta a la variable competencia digital, al ser una teoría de aprendizaje perteneciente a la era digital, la cual estudia la forma en la que se aprende en una sociedad digitalizada articulada en la web, además, el conectivismo, está fundamentado en la conectividad, es decir, en la creación de conexiones, lejos de lo tradicional. Cabe señalar que el conocimiento en la web gravita en la creación, trayendo consigo un cambio de perspectiva y actitud, es un paso de ser solo consumidores de contenidos creados por otras personas a ser los propios productos de conocimiento.

El conectivismo, según Siemens (2006), considera al aprendizaje como un proceso acontecido dentro de entornos difusos de componentes esenciales cambiantes, los cuales no están bajo el control de la persona. Además, está orientado en la conexión de un conjunto de datos especializados, siendo las conexiones de mayor importancia las que facultan la mayor adquisición de conocimientos de los que tenemos en la actualidad, orientado en la constante adquisición de información nueva y en la habilidad de distinguir información relevante como la que no resulta vital.

En cuanto a la competencia digital, Lévano-Francia et ál. (2019), dan a entender que contiene amplio significado respecto a su aplicación en el campo educativo, cuyo espectro influye en el campo del aprendizaje, investigación, recreación y sociedad, además aportan perspectivas de empoderamiento, así como presencia de las nuevas predisposiciones 
culturales y de diversión del presente siglo. Asimismo, la competencia digital fue definida como el manejo con actitud crítica y responsable de herramientas digitales que son utilizadas para aprender, trabajar y ser participe socialmente, (Consejo de la Unión Europea, 2018 y Lordache et ál., 2017), en tal sentido, adquirir competencias que están apoyadas en el uso de dispositivos digitales como ordenadores, laptops, tabletas, entre otros, facilita la demostración de destrezas básicas referidas a la recuperación, la evaluación, el almacenamiento, la producción, presentación e intercambio de información por medio de entornos digitales del internet.

Desde la perspectiva de Lund et ál. (2014), la competencia digital docente incluye la capacidad de recolectar y analizar información basada en actividades de aprendizaje online resueltas por los escolares, también la de conectarlos a experiencias de aprendizaje desde cualquier lugar, en cualquier momento a través de dispositivos digitales facilitando entornos de aprendizaje los cuales tienen como propósito que los estudiantes aprendan de experiencia y conocimientos de otros fomentando la búsqueda de información responsable y veraz, asimismo, desde la perspectiva de Zabala et ál. (2016), la competencia digital es la adquisición de capacidades sobre el uso adecuado de herramientas tecnológicas de forma creativa y crítica, un insumo para el enriquecimiento del trabajo pedagógico del docente el cual forma parte de su crecimiento profesional en el ámbito educativo en donde se desenvuelve. En tanto, From (2017), definió a la competencia digital docente como la virtud de adaptar con consistencia su trabajo pedagógico, como también a evaluar y revisar el proceso de enseñanza coadyuvada por el uso de tecnología, con miras a brindar reforzamiento para consolidar el aprendizaje de los escolares, además envuelve toda labor docente en la que es necesario el uso de recursos tecnológicos.

El MINEDU (2016), por otro lado, lo consideró indispensable en la formación del profesorado, al tenerlo como base en la identificación y uso de recursos tecnológicos, que promueven la búsqueda de conocimiento, comunicación y colaboración, esperando que generen innovación enriqueciendo su labor docente por medio del desarrollo de habilidades digitales asistido por las tecnologías actuales. Además, según Foulger et ál. (2017), es necesario que el docente conozca sobre tecnología digital y que demuestre estar apto para utilizarlas de forma segura, crítica y creativa, cuya intención está basada en la mejora de su proceso de enseñanza y por ende en la mejora del aprendizaje de los 
escolares.

En tal sentido, Rokenes y Jrumsvik (2014), sostienen que la competencia digital involucra una vasta variedad de habilidades, así como un vasto conocimiento sociológico que permite el uso de entornos digitalizados de forma eficiente, cabe señalar que la intensidad que sostiene la adquisición del pensamiento crítico constituye un trozo importante de la competencia digital, reconocida por Instefjord (2015), quien hace énfasis en el juicio y reflexión del uso de recursos tecnológicos sobre la edificación de nuevos aprendizajes.

Además, la UNESCO (2019), mencionó que integrar eficazmente recursos tecnológicos en su práctica docente dependerá de lo capaz que es para estructurar su enseñanza, combinando de forma adecuada lo tecnológico con lo pedagógico. Es así que se destaca la importancia que la adaptación actual que hacen los docentes demanda el fortalecimiento de la competencia digital, con el de fortalecer los canales de aprendizaje. De acuerdo al Instituto Nacional de Tecnología Educativas y de Formación del Profesorado, INTEF (2017), se detallan cinco dimensiones que todo docente debe poseer para desarrollar su competencia digital:

Como primera dimensión, información y alfabetización informacional, basada en la búsqueda sistemática de información, datos y contenido digital, además de obtener las habilidades de procesar, comprender y evaluar críticamente la información obtenida, conllevando al almacenamiento de las misma para su fácil recuperación y uso posterior para el trabajo pedagógico docente (INTEF, 2017).

Como segunda dimensión, comunicación y colaboración, competencia basada en la interacción mediada por la tecnología digital, además de compartir información y contenido digital, facilidad de conectarse y colaborar con otros docentes haciendo uso de herramientas digitales, interacción y participación en comunidades digitalizadas tomando en cuenta normas conductuales de respeto mutuo (netiqueta), conciencia intercultural y que gestión de su propia identidad digital (INTEF, 2017).

Como tercera dimensión, creación de contenido digital, competencia basada en la producción y edición de contenido digital reciente, integrando y reelaborando saberes y contenidos anteriores, realizando producción artística, además de crear contenido multimedia y programación informática y en el saber sobre los derechos de autor y licencias para poder aplicarlas con seguridad (INTEF, 2017).

Como cuarta dimensión seguridad, basada en el resguardo de datos e información 
personal, protección de su propia identidad digital, del contenido digital, es la toma de medidas que le brinden seguridad y al uso con un alto grado de responsabilidad y seguro de las herramientas tecnológicas (INTEF, 2017).

Y como quinta dimensión resolución de problemas, basada en la identificación de necesidades sobre el manejo de recursos digitales, el decidir de manera informada respecto a herramientas digitales que más se ajusten al objetivo planteado, resolver problemas por medio del uso de herramientas digitales, uso de la tecnología de manera inventiva, capacidad para resolver posibles problemas técnicos, además de poder actualizar su propia competencia y asimismo la de otras (INTEF, 2017).

\section{ESTRATEGIAS METODOLÓGICAS}

Tipo de investigación: El presente estudio fue de tipo aplicado, porque tuvo como propósito solucionar la problemática sobre la competencia digital docente en tres instituciones educativas, enfocándose en buscar y consolidar el conocimiento para su aplicación. directamente, Al respecto, Lozada, (2014), señaló que la investigación de tipo aplicada busca generar conocimiento con una directa aplicación en una realidad determinada, apoyado del conocimiento de la investigación de tipo básico.

Enfoque de investigación: Además, fue de enfoque cuantitativo, porque en este estudio se utilizó procedimientos de análisis descriptivos e inferenciales, en tal sentido Hernández et ál. (2014), mencionaron que dicho enfoque hace uso del recojo de información con el propósito de probar supuestos basados en la medición numérica y el posterior análisis haciendo uso de la estadística.

Método de investigación: De método hipotético-deductivo, ya que al inicio se observó el problema sobre la competencia digital docente que permitió plantear hipótesis para explicar dicha problemática, deduciendo sus consecuencias elementales de la propia hipótesis verificándolo por medios deductivos. Asimismo, Pimienta y de la Orden (2017), mencionaron que este método permite la estructuración de razonamientos de los que se infieren u obtienen conclusiones desde una o varias hipótesis.

Nivel de investigación: De nivel descriptivo-comparativo, al haber recolectado información de tres instituciones educativas con el propósito de observar su manera de comportarse respecto de la competencia digital docente para posteriormente ser comparado dicho comportamiento entre ellos mismos, en tal sentido, HernándezSampieri y Mendoza (2018), es de nivel descriptivo-comparativo al pretender dar a 
conocer las particularidades, perfiles, procesos, propiedades, niveles o cualquier otra manifestación que sea sometida a un análisis de una muestra determinada.

Diseño de investigación: Por otro lado, el diseño fue el no-experimental, al no realizar manipulación alguna de la variable competencia digital docente, siendo solo observado lo acontecido en su estado natural para posteriormente ser analizado, cabe señalar que Hernández et ál. (2014), consideraron al diseño como un plan desarrollado con el propósito de recoger información requerida en un estudio el cual responde a los planteamientos propuestos. A continuación, se muestra el diseño de la investigación Adaptado de Hernández et ál. (2014, p.157).

Figura 1. Diseño

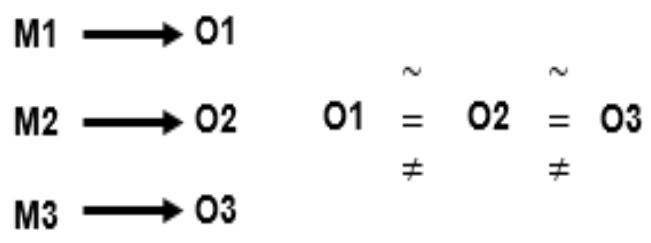

Donde: M1, M2 y M3 se refieren a los docentes de las tres instituciones educativas, O1, $\mathrm{O} 2$ y $\mathrm{O} 3$ se refiere a las observaciones realizadas a cada muestra y los símbolos $\sim,=\mathrm{y} \neq$, son semejante, igual y desigual respectivamente.

Población y muestra. La población de estudio fue conformada por 108 docentes pertenecientes a tres instituciones educativas de nivel secundario de la UGEL Chincheros, Apurímac. Hernández-Sampieri y Mendoza (2018), indicaron que el conjunto finito de casos concordantes con determinadas característicos en un mismo lugar y tiempo es conocido como población.

Tabla 1. Población de estudio

\begin{tabular}{llc}
\hline Distrito & Institución educativa & N. $^{\circ}$ de docentes \\
\hline Chincheros & Túpac Amaru & 33 \\
Uripa & José María Arguedas & 51 \\
Ranracancha & Los libertadores & 24
\end{tabular}

Total 108

$\overline{\text { Para poder definir la muestra, se usó el muestreo probabilístico estratificado, hallando en }}$ primer lugar el tamaño muestral apoyado por la fórmula finita, evidenciando que el tamaño de la muestra es de 85 docentes, posteriormente se halló el factor proporcional para mantener la representatividad de cada institución educativa, en tal sentido se dividió 
el tamaño muestral (85), con el tamaño poblacional (108), obteniendo 0,787 ; de factor proporcional el cual será multiplicado por la cantidad de docentes de cada institución educativa y de esa manera obtener la muestra.

Tabla 2. Muestra de estudio

\begin{tabular}{lccc}
\multicolumn{1}{c}{ Institución educativa } & Población & Factor & Muestra \\
\hline Túpac Amaru & 33 & 0.787 & 26 \\
José María Arguedas & 51 & 0.787 & 40 \\
Los libertadores & 24 & 0.787 & 19 \\
\hline \multicolumn{1}{c}{ Total } & $\mathbf{1 0 8}$ & & $\mathbf{8 5}$ \\
\hline
\end{tabular}

Técnicas e instrumentos. Para el recojo de la información necesaria el cual dio respuesta a los problemas de investigación anteriormente planteados, se seleccionó como técnica a la encuesta, caracterizada por su practicidad al ser aplicada en un solo momento en donde la información es obtenida mediante preguntas, además se diferencia al no establecer un diálogo directo con las personas entrevistadas siendo su grado de interacción muy bajo (Carhuancho et ál., 2019). Y como instrumento al cuestionario que según Páramo (2017), es la más usada en las ciencias sociales, al permitir la recolección de una basta cantidad de información, además, el recojo de datos se realizó por medio de la colaboración de las personas que se fijó encuestar, valiéndose de ítems estructurados respecto a los indicadores que son precisados al definir las dimensiones que a su vez se desglosan de la variable.

Validez y confiabilidad. La validez del instrumento elaborado para el presente estudio es de contenido, al evidenciar un dominio especial referente al contenido de la variable que se va a medir, para lo cual se sometió a juicio de expertos, por parte de docentes especializados en el área de las ciencias sociales. Asimismo, Vara (2015), la definió como el grado real del instrumento al evidenciar si mide lo que debe medir, dicha afirmación se logra al momento de demostrar que el instrumento muestra la definición abstracta por medio de sus indicadores. La confiabilidad se evidenció al utilizar el estadístico alfa de Cronbach en los datos recolectados de los 85 docentes de tres instituciones educativas de Chincheros, los cuales permitieron concluir que es instrumento que mide a la variable competencia digital docente es confiable con un 0,870 de coeficiente de fiabilidad. En tal sentido, Hernández-Sampieri y Mendoza (2018), dan a entender que para que un instrumento sea fiable debe evidenciar el grado de consistencia respecto al puntaje 
obtenido por un similar conjunto de personas al someterlos a una serie de mediciones haciendo uso del mismo instrumento.

\section{RESULTADOS Y DISCUSIÓN}

\section{Resultados descriptivos:}

Tabla 3. Niveles de la variable competencia digital docente

\begin{tabular}{lcccccc}
\hline \multirow{2}{*}{ Nivel } & \multicolumn{6}{c}{ Instituciones Educativas } \\
\cline { 2 - 7 } & \multicolumn{2}{c}{ Túpac Amaru } & José María Arguedas & \multicolumn{2}{c}{ Los Libertadores } \\
\cline { 2 - 7 } & $\mathbf{f}$ & $\mathbf{\%}$ & $\mathbf{f}$ & $\mathbf{\%}$ & $\mathbf{f}$ & $\%$ \\
\hline Básico & 0 & 0.0 & 0 & 0 & 0 & 0 \\
Intermedio & 4 & 15.4 & 16 & 40.0 & 4 & 21.1 \\
Avanzado & 22 & 84.6 & 24 & 60.0 & 15 & 78.9 \\
\hline Total & $\mathbf{2 6}$ & $\mathbf{1 0 0 . 0}$ & $\mathbf{4 0}$ & $\mathbf{1 0 0 . 0}$ & $\mathbf{1 9}$ & $\mathbf{1 0 0 . 0}$ \\
\hline
\end{tabular}

Figura 2. Gráfico de barras de la variable competencia digital docente

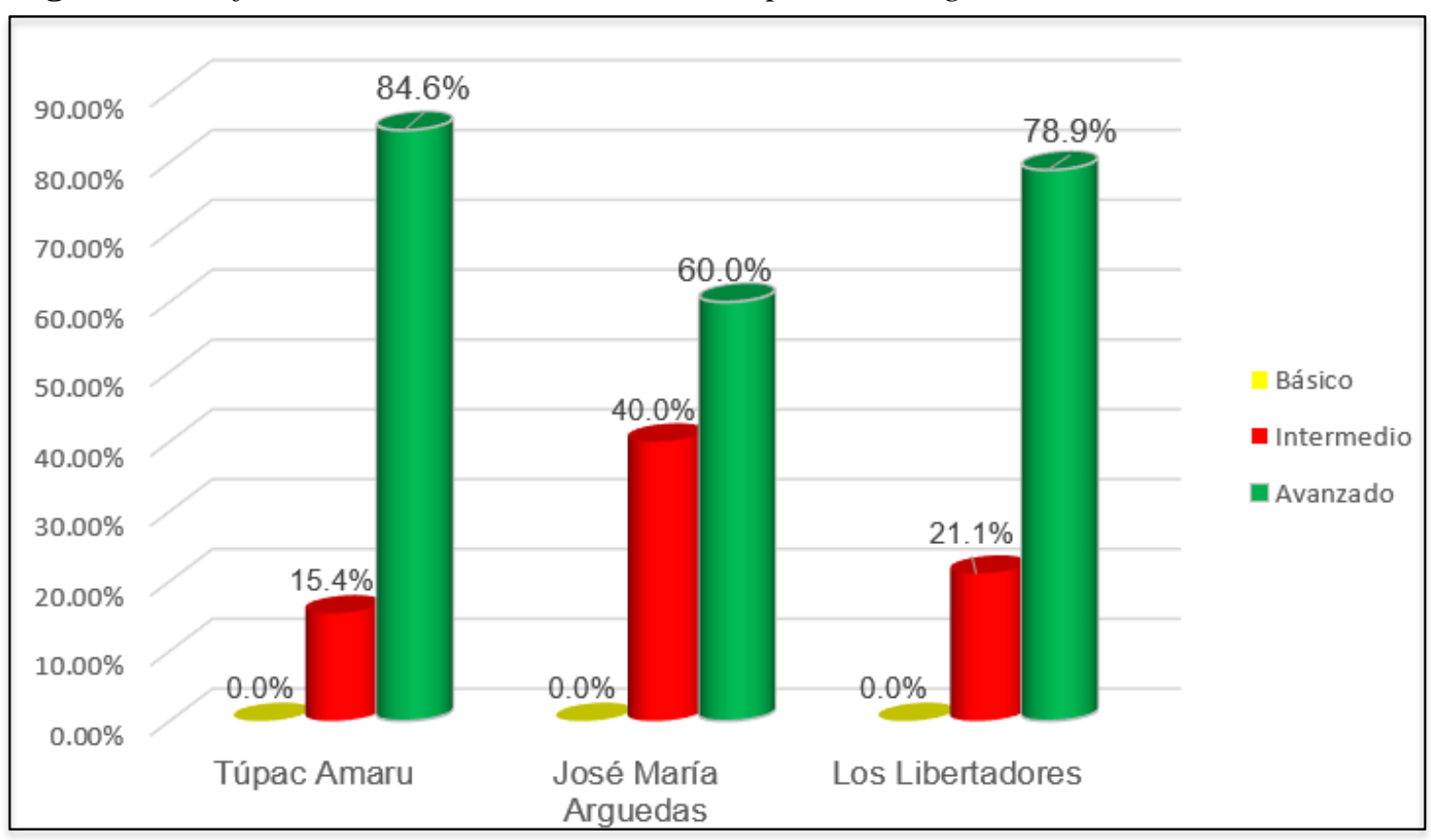

Interpretación: De la tabla 1 y figura 1, se puede deducir en cuanto a la institución educativa Túpac Amaru conformada por una muestra de 26 docentes, se identificó que un $15.4 \%$ se encuentra en el nivel intermedio, el $84.6 \%$ se encuentra en el nivel avanzado, en caso de la institución educativa José María Arguedas tiene como muestra a 40 docentes, se identificó que un $40 \%$ se encuentra en el nivel intermedio, el $60 \%$ se encuentra en nivel avanzado y por último, en la institución educativa Los Libertadores 
que tiene una muestra de 19 docentes, se identificó que un $21.1 \%$ se encuentra en nivel intermedio y el $78.9 \%$ se ubica en el nivel avanzado.

\section{Resultados inferenciales:}

Tabla 4. Prueba de rango promedio y de Kruskal-Wallis

\begin{tabular}{llccccc}
\cline { 2 - 7 } & \multicolumn{1}{c}{$\begin{array}{c}\text { Instituciones } \\
\text { educativas }\end{array}$} & $\mathbf{N}$ & $\begin{array}{c}\text { Rango } \\
\text { promedio }\end{array}$ & $\begin{array}{c}\text { H de Kruskal- } \\
\text { Wallis }\end{array}$ & gl. $\begin{array}{c}\text { Sig. } \\
\text { Asintótica }\end{array}$ \\
\hline Competencia & Túpac Amaru & 26 & 48,46 & & & \\
digital & José María Arguedas & 40 & 38,00 & 5,272 & 2 & \multirow{2}{*}{072} \\
docente & Los Libertadores & 19 & 46,05 & & & \\
\cline { 2 - 7 } & \multicolumn{1}{c}{ Total } & $\mathbf{8 5}$ & & & & \\
\hline
\end{tabular}

Interpretación: El nivel de significancia de la prueba de Kruskal-Wallis fue de 0.072>0.05; además, se observó que la diferencia de rangos en la competencia digital docente, no muestra grandes diferencias, siendo el rango promedio de cada institución educativa de 48,46, 38,00 y 46,05, en tal sentido se tomó la decisión de no rechazar la Ho, por tanto, se concluyó que no existen diferencias significativas en el nivel de competencia digital en docentes de tres instituciones educativas de nivel secundario.

\section{Discusión}

Los resultados que se obtuvo del estudio referente al nivel de competencia digital docente en tres instituciones educativas de nivel secundario, UGEL Chincheros. Apurímac 2021, los datos descriptivos indicaron, que estos tres grupos se ubican en el nivel avanzado de competencia digital con $84.6 \%, 78.9 \%$ y un $60.0 \%$, así mismo la prueba de KruskalWallis con $\mathrm{p}$ - valor $=0.072>0.05$ refiere que no existe diferencias significativas entre estas tres instituciones educativas, este resultado es consecuencia de que el estudio se hizo un año después y durante este tiempo los docentes participaron en diferentes cursos sobre desarrollo de competencia digital; desplegando capacidades sobre el uso adecuado de las herramientas tecnológicas de forma creativa y crítica, considerándose insumo para el trabajo pedagógico y desarrollo profesional en el contexto educativo. Zabala et ál. (2016) así mismo la corriente filosófica del conectivismo, refiere que el aprendizaje se da en un ambiente colaborativo e intercambio de información. Siemens (2006). Estos hallazgos son superiores a los de Zuñiga (2020), quién señala que un 55\% de docentes se encuentra en un nivel bajo y un $35 \%$ se encuentra en nivel medio en competencias digitales. 


\section{CONCLUSIÓN O CONSIDERACIONES FINALES}

Se probó que no existe diferencias significativas en el nivel de competencia digital docente en tres instituciones educativas de nivel secundario. Apurímac, 2021, evidenciándose con los valores perceptuales descriptivos de las tres instituciones educativas. Asimismo, la prueba de Kruskal-Wallis con p-valor mayor al margen de error, indicaron no rechazar la hipótesis nula.

\section{LISTA DE REFERENCIAS}

Cantián, F. (2020, 4 de abril). ¿A qué modelo educativo lleva la Covid-19? La Vanguardia.

https://www.lavanguardia.com/participacion/cartas/20200404/48283729781/deb ate-modelo-educativo-presencial-a-distancia-virtual-pandemia-covid-19.html

Carhuancho, I. M., Nolazco, F. A., Sicheri, L., Guerrero, M. A., y Casana, K. M. (2019). Metodología para la investigación holística. (1ra. Edición). UIDE. https://repositorio.uide.edu.ec/bitstream/37000/3893/3/Metodolog\%c3\%ada\%20 para\%20la\%20investigaci\%c3\%b3n\%20hol\%c3\%adstica.pdf

Consejo de la Unión Europea. (2018). Information and Notices. Official Journey of the European Union, 61(C189), 1-13. https://eur-lex.europa.eu/legalcontent/EN/TXT/?uri=uriserv:OJ.C_.2018.189.01.0001.01.ENG

Cruz, M. A., y Carcausto-Calla, Wilfredo (2020). Digital Competence, Nwe Perspectives for Teaching in Contexts of Social Isolation. Revista EDUSER, 7(2), 119-126. https://doi.org/10.18050/eduser.v7i2.2662

Foulger, T.S., Graziano, K.J., Schmidt-Crawford, D. \& Slykhuis, D.A. (2017). Teacher Educator Technology Competencies. Journal of Technology and 46 Teacher Education, 25(4), 413-448. https://www.learntechlib.org/primary/p/181966/

From, J. (2017). Pedagogical Digital Competence-Between Values, Knowledge and Skills. Higher Education Studies, 7(2). http://doi.org/10.5539/hes.v7n2p43

Gil, J. L., León, J. L., y Morales, M. (2017). Los paradigmas de investigación educativa, desde una perspectiva crítica. Revista Conrado, 13(58), 72-74. https://conrado.ucf.edu.cu/index.php/conrado/article/view/476/510

Hernández, R., Fernández, C. y Baptista, P. (2014). Metodología de la investigación (6ta. Edición). McGraw-Hill. https://www.uca.ac.cr/wpcontent/uploads/2017/10/Investigacion.pdf 
Hernández-Sampieri, R., y Mendoza, C. P. (2018). Metodología de la investigación: las rutas cuantitativas, cualitativas $\mathrm{y}$ mixtas. Mc Graw Hill Education. https://www.panamericana.com.co/metodologia-investigacion-rutas-de-inves$571174 / \mathrm{p}$

INEI (2019). Estadísticas de las Tecnologías de Información y Comunicación en los Hogares. Instituto Nacional de Estadística e Informática. https://www.inei.gob.pe/media/MenuRecursivo/boletines/ticdiciembre.pdf

Instefjord, E. (2015). Appropriation of Digital Competence in Teacher Education. Nordic $\begin{array}{llll}\text { Journal of } & \text { Digital }\end{array}$ https://www.researchgate.net/publication/284901008_Appropriation_of_Digital _Competence_in_Teacher_Education

INTEF. (2017). Marco común de competencia digital docente. Instituto Nacional de Tecnologías Educativas y de Formación del Profesorado. http://aprende.intef.es/sites/default/files/2018-05/2017_1020_MarcoCom\%C3\%BAn-de-Competencia-Digital-Docente.pdf

Levano-Francia, L., Sanchez, S., Guillén-Aparicio, P., Tello-Cabello, S., Herrera-Paico, N., y Collantes-Inga, Z. (2019). Digital Competences and Education. Propósitos y Representaciones, 7(2), 569-588. http://dx.doi.org/10.20511/pyr2019.v7n2.329 Lordache, C., Marien, I., \& Baelden, D. (2017). Developing Digital Skills and Competence quickScan analysis of 13 Digital Literacy Models. Italian Journal of Sociology of Education, 9(1), 6-30. http://ijse.padovauniversitypress.it/2017/1/2

Lozada, J. (2014). Investigación aplicada. Definición, propiedad intelectual e industria. CienciAmérica: Revista de divulgación científica de la Universidad Tecnológica Indoamérica, $3(1)$, $47-50$. https://dialnet.unirioja.es/servlet/articulo?codigo=6163749

Lund,A., Furberg, A., Bakken, J., y Engelien, K. (2014). What does profesional digital competence mean in teacher education?. Nordic journal of digital literacy. 9(6). https://www.idunn.no/dk/2014/04/what_does_professional_digital_competence_ mean_inteacher_e

Marza, M., y Cruz, E. (2018). Gaming como Instrumento Educativo para una Educación en competencias Digitales desde los Academic Skills Centres. Revista General de Información y Documentación, $28(1)$ 45-60. 
http://dx.doi.org/10.5209/RGID.60805

MINEDU. (2016). Estrategia nacional de las tecnologías en la educación básica 20162021- De las TIC a la inteligencia digital. https://cdn.www.gob.pe/uploads/document/file/110893/_505-2016-MINEDU__13-12-2016_07_25_15_-RSG_N_505-2016-MINEDU.pdf

MINEDU. (2020). R.M. Nº160-2020-MINEDU. Disponen el inicio del año escolar a través de la implementación de la estrategia denominada "Aprendo en casa", a partir del 6 de abril de 2020 y aprueban otras disposiciones. https://www.gob.pe/institucion/minedu/normas-legales/466108-160-2020minedu

Páramo, P. (2017). La investigación en ciencias sociales: Técnicas de recolección de información. (1ra. Edición). Universidad Piloto de Colombia.

PCM (2020). Decreto Supremo N 046-2020-PCM que declara el Estado de Emergencia Nacional, por las graves circunstancias que afectan la vida de la Nación a $\begin{array}{lllll}\text { consecuencia del brote del } & \text { COVID }\end{array}$ https://www.gob.pe/institucion/pcm/normas-legales/462244-046-2020-pcm

Pérez, J. (2015). The positivism and the scientiic research. Revista Empresarial ICE, 9(3), 29-34. https://dialnet.unirioja.es/servlet/articulo?codigo=6419741

Pimienta, J. G. y de la Orden, A. (2017). Metodología de la investigación. (3ra. Edición). Pearson Educación de México, http://repositorio.uasb.edu.bo:8080/bitstream/54000/1268/1/PimientaMetodolog\%C3\%ADa\%20de\%201a\%20investigaci\%C3\%B3n\%203ra\%20ed.pd $\mathrm{f}$

Rokenes, F. M., \& Krumsvik, R. (2014). Development of Student Teachers' Digital Competence in Teacher Education - A Literature Review, Nordic Journal of Digital Literacy, 250-280. https://www.researchgate.net/publication/269222866_Development_of_Student _Teachers'_Digital_Competence_in_Teacher_Education__A_Literature_Review

Siemens, G. (2004) Connectivism: a theory for the digital age' eLearningSpace.

UNESCO. (2019). Marco de competencias de los docentes en materia de TIC. https://unesdoc.unesco.org/ark:/48223/pf0000213475 
UNESCO. (2020), Educación en crisis, desafíos del camino a seguir. https://es.unesco.org/news/educacion-crisis-desafios-del-camino-seguir

UNESCO. (2021). La UNESCO revela una pérdida aproximada de dos tercios de un año académico en todo el mundo debido a los cierres de la COVID-19. https://es.unesco.org/news/unesco-revela-perdida-aproximada-dos-tercios-anoacademico-todo-mundo-debido-cierres-covid-19

Vara, A. (2015). 7 Pasos para elaborar una tesis. Lima: Editorial Macro E.I.R.L. https://www.administracion.usmp.edu.pe/investigacion/files/7-PASOSPARAUNA-TESIS-EXITOSA-Desde-la-idea-inicial-hasta-lasustentaci\%C3\%B3n.pdf

Zabala, D., Muñoz, K., y Lozano, E. (2016). Un enfoque de las competencias digitales de los docentes. Revista publicando, 3 (9), 330-340. https://dialnet.unirioja.es/servlet/articulo? $\operatorname{codigo}=5833540$

Zuñiga, J. F. (2020). Competencias digitales en docentes de una institución educativa, San Camilo, Ecuador 2019. [Tesis de maestría, Universidad César Vallejo]. https://hdl.handle.net/20.500.12692/43169 